\title{
Estado nutricional de crianças e serviços de saúde prestados por equipes de Saúde da Família
}

\author{
Nutritional status of children and health services \\ provided by Family Health teams
}

Dixis Figueroa Pedraza (http://orcid.org/0000-0002-5394-828X) 1,2 $^{1,2}$

Maria Mônica de Oliveira (https://orcid.org/0000-0002-2775-4318) ${ }^{3}$

${ }^{1}$ Universidade Estadual da Paraíba. Av. das Baraúnas 351 Campus I, Bodocongó. 58109-753 Campina Grande PB Brasil.

dixisfigueroa@gmail.com

${ }^{2}$ Programa de PósGraduação em Saúde da Família, Rede Nordeste de Formação em Saúde da Família. Campina Grande PB Brasil.

${ }^{3}$ Centro de Ensino Grau

Técnico. Campina Grande PB Brasil.

\begin{abstract}
The scope of this article is to analyze if the nutritional status of children is associated with the health services offered by Family Health teams. This involves a cross-sectional study carried out in areas covered by the Family Health Strategy in two cities in the State of Paraiba, with a representative and random sample of 324 children. Linear regression was used to analyze the association of sociodemographic and health services characteristics with $z$-score height/age, weight/ age and body mass/age anthropometric indices. Among the 319 children observed, there was a predominance of beneficiaries of the Bolsa Familia Program and with inadequate use of the Child Health Handbook. The average of the weight/age $z$-score was lower in children from understaffed health teams ( $p=0.02$ ), without a nutritionist $(p=0.03)$ and with unqualified professionals in the areas of food and nutrition $(p=0.04)$. The$r e$ was an average increase of 1.43 standard deviation in height/age in the case of the adequate application of the Child Health Handbook. This study contributes to the knowledge by indicating associations of the nutritional status of the child with structural (human resources) and procedural (adherence to the Child Health Handbook) characteristics of health services.
\end{abstract}

Key words Primary health care, Evaluation of health services, Growth
Resumo O objetivo deste artigo é analisar se o estado nutricional de crianças está associado aos serviços de saúde oferecidos por equipes de Saúde da Família. Estudo transversal realizado em áreas de abrangência da Estratégia Saúde da Família em dois municípios do Estado da Paraíba, com amostra representativa e aleatória de 324 crianças. Utilizou-se regressão linear para analisar a associação das características sociodemográficas das crianças e dos serviços de saúde com os indices antropométricos estatura/idade, peso/idade e massa corporal/idade em escore Z. Nas 319 crianças observadas houve predominio de beneficiárias do Programa Bolsa Família e com uso inadequado da Caderneta de Saúde da Criança. A média de escore $Z$ do peso/idade foi menor nas crianças de equipes de saúde incompletas $(p=0,02)$, sem nutricionista $(p=0,03)$ e com profissionais sem capacitação na área de alimentação e nutrição $(p=0,04)$. Houve um aumento, em média, de 1,43 desvio-padrão de estatura/idade no caso de uso adequado da Caderneta de Saúde da Criança. O presente estudo contribui com o conhecimento ao indicar associações do estado nutricional da criança com características estruturais (recursos humanos) e processuais (preenchimento da Caderneta de Saúde da Criança) dos serviços de saúde.

Palavras-chave Atenção Primária à Saúde, Avaliação de serviços de saúde, Crescimento 


\section{Introdução}

Nos países de América Latina e no Brasil a situação nutricional da população apresentou marcadas mudanças nas últimas décadas ${ }^{1,2}$. $\mathrm{O}$ processo de transição epidemiológica e nutricional tem desencadeado modificações na distribuição geográfica, social e biológica das doenças, com prevalências expressivas de déficit de estatura e excesso de peso $^{1-3}$. O estado nutricional da criança é multicausal ${ }^{3}$, uma vez que os problemas nutricionais são resultados de ambientes sociais e econômicos desfavoráveis que se expressam em limitações no poder aquisitivo, nas condições de saneamento e no acesso aos serviços de saúde $e^{2-4}$.

Para o enfrentamento dos problemas de nutrição é necessário a construção de um entorno favorável, o desenvolvimento de intervenções e programas específicos de nutrição, e a implementação de outras intervenções relacionadas à área que incorporem ações nutricionais vinculadas aos determinantes subjacentes da malnutrição ${ }^{4}$. As intervenções não específicas de nutrição, mas relacionadas a ela, incluem os serviços básicos de saúde como formas essenciais de responder às necessidades de saúde, incluindo ações de cuidado nutricional e promoção da alimentação saudável ${ }^{2,4}$. Serviços de saúde organizados a partir de uma atenção primária à saúde, estruturada em conformidade com os seus atributos, são mais eficazes e de maior qualidade. Os atributos essenciais dessa atenção são o primeiro contato (acessibilidade e uso de serviços para cada novo problema ou para acompanhamento), a longitudinalidade (aporte regular de cuidados), a integralidade (prestação de serviços adequados que atendam às necessidades da população) e a coordenação (capacidade de garantir a continuidade da atenção) $)^{5}$.

O sistema de saúde, sobretudo no nível primário, também é responsável pela avaliação do crescimento, sendo muitas vezes negligenciado devido à diversidade de fatores que incluem a falta de habilidades e de tempo dos profissionais ${ }^{6}$. Além disso, ambas as condições (sobrepeso e desnutrição) devem ser enfrentadas simultaneamente de forma coordenada, mas na maioria das vezes não existem as condições apropriadas para isso ${ }^{1,2}$. No contexto brasileiro, as ações de alimentação e nutrição na atenção básica devem ser operacionalizadas de forma multidisciplinar com foco na vigilância alimentar e nutricional, na promoção da alimentação saudável, na prevenção de agravos relacionados à alimentação e nutrição como as deficiências de micronutrientes e a obesidade e no atendimento das famílias beneficiárias do Programa Bolsa Família (PBF). Para o fortalecimento dessas ações nas equipes de saúde, a inserção do nutricionista no Núcleo Ampliado de Saúde da Família e Atenção Básica (NASF-AB) tem um papel importante, pois possibilita o matriciamento e atuação qualificada desde o diagnóstico da situação de segurança alimentar e nutricional até o atendimento individualizado 7 .

Desprende-se dessa situação a importância de conhecer o impacto das intervenções de saúde no estado nutricional ${ }^{4}$. No Brasil, nesse sentido, pesquisas com foco no PBF têm destacado a ausência de efeitos do Programa no estado nutricional dos beneficiários. Os pesquisadores destacam que essas circunstâncias podem estar relacionadas à qualidade dos serviços de saúde, uma vez que a adesão às condicionalidades é altamente dependente do acesso aos mesmos. Por outro lado, discutem a possibilidade do benefício contribuir com o desenvolvimento de sobrepeso, pois a ajuda recebida pode produzir aumento do consumo de alimentos de maior densidade calórica e baixo valor nutritivo, principalmente nos casos de insegurança alimentar grave ${ }^{8}$. Contudo, a discussão principal em torno da relação entre os serviços de saúde e a situação nutricional adverte que os estudos sobre modelos de intervenção e de avaliação de programas ainda são raros, apesar dos desafios impostos aos serviços básicos de saúde no Brasil por respostas contínuas e oportunas aos problemas de nutrição9.

O objetivo deste estudo foi analisar se o estado nutricional de crianças está associado aos serviços de saúde oferecidos por equipes de Saúde da Família.

\section{Métodos}

Estudo de delineamento transversal conduzido no contexto da Estratégia Saúde da Família (ESF) em dois municípios do Estado da Paraíba. A escolha dos municípios considerou suas semelhanças quanto à posição geográfica (localização na região metropolitana da capital do estado com acesso à rede de serviços nela disponibilizados); grau de urbanização (de quase 100\%); indicadores demográfico-sociais (índice de desenvolvimento humano de nível médio) e tradição na organização de serviços primários de saúde (cobertura populacional da ESF de 100\%).

Um dos municípios tem população de 57.944 habitantes (4.596 são crianças menores de cinco anos) e sistema de saúde composto por 19 equi- 
pes da ESF, e o outro tem população de 99.716 habitantes (7.862 são crianças menores de cinco anos) e sistema de saúde com 28 equipes da ESF. Em ambos os municípios as unidades de saúde da ESF tinham na época do estudo mínimo de dez anos de funcionamento e contemplavam apenas uma equipe. Entretanto, no primeiro município as equipes mínimas de saúde são ampliadas com a inserção de nutricionista, enquanto no outro a atuação desse profissional acontece por meio dos NASF-AB.

Crianças menores de cinco anos constituíram a população de estudo. Tomando-se por base para o cálculo amostral um nível de 95\% de confiança, poder do teste de $80 \%$, relação entre expostos e não expostos à intervenção de $1: 1$, prevalência de desfechos de no mínimo 25\%, 10\% para compensar eventuais perdas, $15 \%$ para controle de fatores de confusão e $60 \%$ para efeito de delineamento, estimou-se a necessidade de estudar 324 sujeitos. Essa amostra é suficiente para todos os desfechos do estudo. A prevalência de sobrepeso/ obesidade usada no cálculo foi a encontrada para um dos municípios de estudo (24,9\%), segundo dados do Sistema de Vigilância Alimentar e Nutricional (SISVAN) de 2013.

A amostra foi selecionada em dois estágios. No primeiro, foram sorteadas aleatoriamente as equipes de Saúde da Família, e no segundo estágio um número fixo de crianças. Em cada município foram selecionadas nove equipes da ESF que representaram no mínimo $1 / 3$ do total das mesmas. Cada equipe contribuiu, em média, com 18 crianças para o total da amostra que obedeceu a dois critérios: 1) uma amostra dos atendimentos nas consultas de puericultura no dia da coleta de dados, em um dia típico de trabalho; 2) uma amostra de crianças encaminhadas aos serviços de saúde pelos Agentes Comunitários de Saúde (ACS), conforme rotina de trabalho. As crianças foram selecionadas aleatoriamente, considerando-se para o segundo momento a quantidade que permitisse completar o total previsto para cada equipe de saúde com base no quantitativo de crianças observadas no primeiro critério.

A coleta foi realizada nas unidades de saúde entre julho e dezembro de 2014 por profissionais e estudantes de graduação e pós-graduação da área de saúde capacitados e supervisionados. Os entrevistadores foram treinados na aplicação do questionário e no manuseio da Caderneta de Saúde da Criança (CSC), com quantidade de sessões suficientes para padronização da coleta de dados. Os dados antropométricos foram obtidos por antropometristas treinados e experientes.
As características sociodemográficas das crianças foram obtidas utilizando-se questionário estruturado aplicado às mães das crianças. A data de nascimento e o sexo foram retirados da CSC. A idade da criança foi calculada em meses, mediante a diferença entre as datas da entrevista e de nascimento.

As informações sobre os serviços de saúde basearam-se na estrutura das unidades de saúde, no uso da CSC e na avaliação materna da assistência. Para caracterização da estrutura das unidades de saúde utilizou-se questionário padronizado com perguntas fechadas, o qual foi respondido com a participação de todos os membros da equipe de saúde, sob a liderança do enfermeiro. Quando necessário, foram realizadas confirmações por meio de observação. Contemplaram-se informações sobre os recursos humanos, a disponibilidade de medicamentos e suplementos, e a disponibilidade de documentos técnicos do Ministério da Saúde. No Quadro 1 encontram-se os itens de análise utilizados para cada uma dessas dimensões e seus critérios de classificação.

O uso da CSC considerou a versão de posse e o preenchimento do instrumento. Com base na idade da criança, a caderneta foi considerada preenchida corretamente se estava com número de registros nos gráficos de crescimento (perímetro cefálico $\mathrm{x}$ idade, peso $\mathrm{x}$ idade, altura $\mathrm{x}$ idade e Índice de Massa Corporal x idade) consoantes com o mínimo de consultas preconizadas pelo Ministério da Saúde ${ }^{10}$, com número de registros de frascos de suplemento de ferro nos quadros de anotações da suplementação preventiva de ferro e vitamina A em conformidade com o preconizado pelo Programa Nacional de Suplementação de Ferro $^{11}$, com número de registros de suplementação com vitamina A nos quadros de anotações da suplementação preventiva de ferro e vitamina A e/ou nos quadros de anotações das vacinas de acordo com as normas do Programa Nacional de Suplementação de Vitamina $\mathrm{A}^{12}$ e com os registros de vacinas nos quadros de anotações para esses fins seguindo as recomendações do Programa Nacional de Imunização ${ }^{13}$. O uso da CSC foi considerado adequado quando da posse da terceira versão e o preenchimento correto de todos ao parâmetros de interesse.

A avaliação materna dos serviços de saúde recebidos foi realizada por meio do Primary Care Assessment Tool (PCATool) ${ }^{14}$, versão crianças, contemplando os componentes acesso de primeiro contato-utilização (três perguntas), acesso de primeiro contato-acessibilidade (seis perguntas), longitudinalidade (14 perguntas) e integra- 
Quadro 1. Variáveis das dimensões da estrutura das unidades de saúde e critérios de classificação considerados na avaliação dos serviços de saúde. Região Metropolitana de João Pessoa, PB, 2014.

\begin{tabular}{|c|}
\hline Dimensões (critérios de classificação) \\
\hline I - Recursos humanos \\
\hline $\begin{array}{l}\text { - Completitude da equipe mínima (sim: presença de médico, enfermeiro, odontólogo, técnico ou auxiliar de } \\
\text { enfermagem e agente comunitário de saúde; não: ausência de qualquer profissional) } \\
\text { - Inserção de nutricionista na equipe de saúde (sim: nutricionista formando parte da equipe de saúde no formato } \\
\text { ampliado; não: nutricionista formando parte do Núcleo de Apoio à Saúde da Família) } \\
\text { - Capacitação dos profissionais de saúde na área de alimentação e nutrição (adequado: médico e/ou enfermeiro } \\
\text { com treinamento sobre a Política Nacional de Alimentação e Nutrição ou na organização das ações de } \\
\text { alimentação e nutrição na Atenção Primária à Saúde/Estratégia Saúde da Família; inadequado: médico e/ou } \\
\text { enfermeiro sem treinamento). }\end{array}$ \\
\hline II - Disponibilidade de medicamentos e suplementos \\
\hline $\begin{array}{l}\text { Disponibilidade de medicamentos e suplementos* (adequado: média de medicamentos e suplementos } \\
\text { disponíveis } \geq 6,6 \text {; inadequado: média de medicamentos e suplementos disponíveis }<6,6 \text { ). } \\
{ }^{*} \text { Medicamentos e suplementos considerados (pontuados com um ponto quando disponíveis e com zero ponto } \\
\text { se não disponíveis): adrenalina, antibiótico oral (suspensão), anticonvulsivante, anti-helmíntico (suspensão), } \\
\text { antitérmico (suspensão), bronco dilatador, medicação tópica (nistatina, permanganato de potássio), sais de } \\
\text { reidratação oral, sulfato ferroso, vitamina A. }\end{array}$ \\
\hline III - Disponibilidade de documentos técnicos do Ministério da Saúde \\
\hline $\begin{array}{l}\text { Disponibilidade de documentos técnicos do Ministério da Saúde* (adequado: média de documentos técnicos } \\
\text { disponíveis } \geq 6,6 \text {; inadequado: média de documentos técnicos disponíveis }<6,6 \text { ). } \\
\text { * Documentos técnicos considerados (pontuados com } 0,5 \text { pontos quando disponíveis e com zero ponto se } \\
\text { não disponíveis): Assistência pré-natal, imunização, cuidado ao recém-nascido saudável, acompanhamento do } \\
\text { crescimento, promoção do aleitamento materno, promoção da alimentação saudável, atendimento da criança } \\
\text { desnutrida, controle das doenças diarreicas, tratamento das infecções respiratórias agudas, Política Nacional de } \\
\text { Alimentação e Nutrição, matriz de ações de alimentação e nutrição na atenção básica à saúde, protocolos do } \\
\text { Sistema de Vigilância Alimentar e Nutricional, norma técnica do Sistema de Vigilância Alimentar e Nutricional, } \\
\text { diretrizes do Núcleo de Apoio à Saúde da Família, guia alimentar para a população brasileira, guia alimentar } \\
\text { para crianças menores de dois anos, manual operacional do programa de suplementação com ferro, manual } \\
\text { operacional do programa de suplementação com vitamina A, manual de orientações do Programa Bolsa Família, } \\
\text { manual de alimentação e nutrição do Programa Bolsa Família para os agentes comunitários de saúde. }\end{array}$ \\
\hline
\end{tabular}

Fonte: Elaborado pelos autores.

lidade-serviços prestados (cinco perguntas). O acesso de primeiro contato, a longitudinalidade e a integralidade constituem três dos quatro atributos essenciais dos serviços de Atenção Primária à Saúde. $\mathrm{O}$ acesso representa a acessibilidade e utilização do serviço de saúde como fonte de cuidado a cada novo problema ou novo episódio de um mesmo problema de saúde; a longitudinalidade, a existência de uma fonte continuada de atenção, assim como sua utilização ao longo do tempo; e a integralidade, o leque de serviços disponíveis e prestados pelo serviço ${ }^{14}$.

Para todas as perguntas, o PCATool apresenta quatro opções de respostas do tipo Likert ${ }^{5}$, com pontuações de 1 a 4 para cada elemento que compõe o atributo: com certeza, sim (4); provavelmente, sim (3); provavelmente, não (2); com certeza, não (1). A análise dos dados se deu conforme recomendação dos organizadores do instrumento. Para cada atributo, os escores cal- culados foram transformados em uma escala contínua, variando entre zero e dez, por meio da fórmula: Escore ajustado $=[($ escore obtido- 1$) /$ (4-1)] $\times 10^{14}$. O atributo foi considerado adequado nos casos de valores de escore $\geq 6,6$ (três ou mais segundo a escala Likert $)^{5}$.

As crianças menores de dois anos tiveram o comprimento medido por meio de antropômetro infantil de madeira (Alturexata ${ }^{\circledR}$ ) com amplitude de $130 \mathrm{~cm}$ e subdivisões de $0,1 \mathrm{~cm}$. As crianças de dois anos ou mais tiveram a altura medida por meio de estadiômetro (WCS ${ }^{\circledR}$ ) com amplitude de $200 \mathrm{~cm}$ e subdivisões de $0,1 \mathrm{~cm}$. As medições foram realizadas em duplicata, com a finalidade de se obter a média dos valores, sendo que a diferença entre elas não deveria superar 0,3 mm (caso ultrapassa-se uma terceira medida era tomada). Todas as crianças foram pesadas utilizando balança eletrônica do tipo plataforma com capacidade para 150 quilogramas e graduação 
em 100 gramas (Tanita UM-080 ${ }^{\circledR}$ ). Na pesagem, foi permitida apenas uma peça íntima leve, e no caso de crianças, foram retiradas as fraldas das que usavam. O peso de crianças de colo foi calculado pela diferença entre o peso da mãe com a criança no colo e o peso da mãe. As medições foram realizadas de acordo com normas técnicas padronizadas, obedecendo aos procedimentos recomendados pela Organização Mundial da Saúde (OMS) ${ }^{15}$.

Os desfechos do presente estudo foram os índices estatura/idade, peso/idade e índice de massa corporal/idade em escore $Z$, tendo como referência o padrão de crescimento da $\mathrm{OMS}^{16}$. Observou-se a distribuição das médias dos três índices antropométricos de acordo com as características sociodemográficas das crianças e dos serviços de saúde, com a finalidade de detectar diferenças entre os grupos.

As variáveis independentes utilizadas nas análises foram: sexo da criança (masculino, feminino); idade da criança (24-60 meses, $<24$ meses); escolaridade materna (ensino fundamental completo ou superior, sem instrução ou ensino fundamental incompleto); esgotamento sanitário do domicílio (rede pública, outros); benefício do PBF (sim, não); completitude da equipe mínima de saúde (sim, não); inserção de nutricionista na equipe de saúde (sim, não); capacitação dos profissionais de saúde na área de alimentação e nutrição (adequado, inadequado); disponibilidade de medicamentos e suplementos na equipe de saúde (adequado, inadequado); disponibilidade de documentos técnicos do Ministério da Saúde na equipe de saúde (adequado, inadequado); uso da CSC (adequado, inadequado); utilização dos serviços de saúde (adequado, inadequado); acessibilidade aos serviços de saúde (adequado, inadequado); longitudinalidade dos serviços de saúde (adequado, inadequado); integralidade dos serviços de saúde (adequado, inadequado). $\mathrm{O}$ modelo teórico utilizado encontra-se na Figura 1.

$\mathrm{Na}$ análise bivariada, avaliou-se se as médias dos índices antropométricos em escore $\mathrm{Z}$ eram diferentes entre as categorias das variáveis relacionadas às características sociodemográficas das crianças e dos serviços de saúde, por meio do teste $t$-student. As variáveis que apresentaram p-valor inferior a 0,2 , nos testes acima citados, foram selecionadas para a análise multivariável realizada pela regressão linear múltipla a fim de identificar as características associadas a variações nos índices antropométricos.

Para a regressão linear múltipla utilizou-se modelo hierarquizado em quatro níveis para controle de variáveis de confusão: primeiro nível - variáveis sociodemográficas; segundo nível variáveis relacionadas à estrutura dos serviços de saúde; terceiro nível - variáveis relacionadas ao processo de trabalho dos profissionais de saúde (uso da CSC); quarto nível - variáveis relacionadas ao resultado da oferta dos serviços de saúde (avaliação materna da assistência). As análises foram realizadas utilizando o processo retrógrado de seleção, pelo módulo de passos. Para análises subsequentes foram mantidas as variáveis que permaneceram associados após controlados fatores de confundimento do mesmo nível e os de níveis hierárquicos superiores. Os resultados foram apresentados por meio do coeficiente de regressão (beta) e seus respectivos intervalos de confiança de 95\% (IC95\%). Foi estabelecido o critério de significância estatística $\mathrm{p}<0,05$. As análises foram conduzidas no pacote estatístico Stata versão 11.0.

Este estudo foi aprovado pelo Comitê de Ética em Pesquisa da Universidade Estadual da Paraíba em 17/12/2013. As mães/responsáveis das crianças que participaram do estudo assinaram o Termo de Consentimento Livre e Esclarecido.

\section{Resultados}

Das 324 crianças selecionadas para o estudo, 319 tiveram os dados antropométricos devidamente obtidos e foram contempladas nas análises. Observou-se que $51,1 \%$ das crianças eram do sexo masculino e 74,9\% menores de 24 meses. A maioria das crianças tinha mães com Ensino Fundamental completo ou Superior (79,0\%), residia em domicílios sem rede pública de esgoto $(80,9 \%)$ e era beneficiária do PBF $(59,6 \%)$. Segundo a avaliação da estrutura das unidades de saúde, apenas 54 das crianças observadas residiam em áreas de abrangência de três equipes de saúde cuja disponibilidade de documentos técnicos do Ministério da Saúde foi considerada adequada. O uso da CSC foi adequado em 16,9\% das crianças. Segundo a avaliação materna da assistência, os serviços prestados para as crianças foram inadequados em $42,3 \%$ para a utilização, $70,8 \%$ na acessibilidade, $85,9 \%$ referido à longitudinalidade e $75,9 \%$ no quesito integralidade (Tabela 1).

Os valores médios de estatura/idade, peso/ idade e índice de massa corporal/idade em escores $Z$ segundo as variáveis de exposição encontram-se disponíveis na Tabela 1. Observa-se que as médias do peso/idade e do índice de massa 


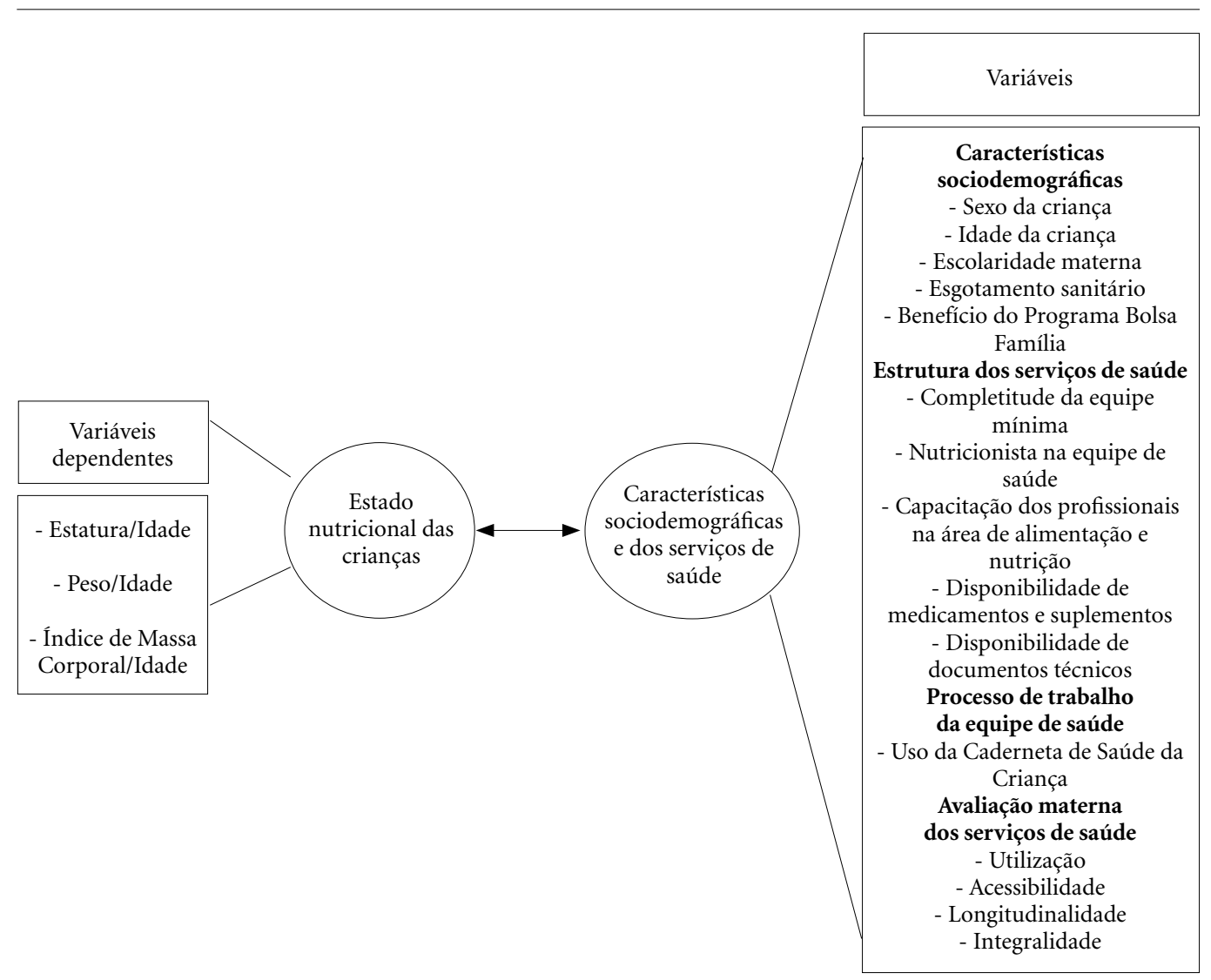

Figura 1. Modelo teórico utilizado para a análise entre o estado nutricional das crianças e as características sociodemográficas e dos serviços de saúde. Região Metropolitana de João Pessoa, PB, 2014.

Fonte: Elaborado pelos autores.

corporal/idade, ao contrário do que foi observado para a estatura/idade, foram todas positivas, independente das características sociodemográficas das crianças e dos serviços de saúde. A média do peso/idade foi menor nas equipes de saúde sem todos os profissionais da equipe mínima $(\mathrm{p}=0,02)$, sem nutricionista $(\mathrm{p}=0,03)$ e com profissionais sem capacitação na área de alimentação e nutrição $(p=0,04)$. Em relação à estatura/idade, nota-se que o uso adequado da CSC representou um aumento, em média, de 1,43 desvio-padrão (variação entre -1,11 e -0,32).

As análises dos efeitos combinados das variáveis exploratórias em relação aos escores Z de estatura/idade, peso/idade e índice de massa corporal encontra-se na Tabela 2. Segundo os modelos de regressão dos desfechos, foi possível observar que o índice peso/idade permaneceu associado negativamente às mesmas características das equipes de saúde registradas nas análises bivaria- das: incompletitude da equipe mínima de saúde $(p=0,02)$, ausência de nutricionista na equipe de saúde $(\mathrm{p}=0,03)$ e profissionais não capacitados na área de alimentação e nutrição $(p=0,04)$. Em relação à estatura/idade, além do uso inadequado da CSC que manteve a associação da análise não ajustada, a escolaridade materna (mãe sem instrução ou com Ensino Fundamental incompleto) também influenciou de forma negativa a condição nutricional da criança.

\section{Discussão}

O principal resultado desse estudo aponta que crianças atendidas em serviços de saúde deficientes tiveram piores índices nutricionais. Tal constatação está em consonância com a literatura, visto que a disponibilidade de força de trabalho em saúde e sua qualificação são componentes 
Tabela 1. Média e intervalo de confiança de 95\% de estatura/idade, peso/idade e índice de massa corporal/ idade em escore $\mathrm{Z}$ de acordo com características sociodemográficas das crianças e dos serviços de saúde. Região Metropolitana de João Pessoa, PB, 2014 ( $\mathrm{N}=319)$.

\begin{tabular}{|c|c|c|c|c|c|c|c|}
\hline \multirow{2}{*}{ Variáveis } & \multirow{2}{*}{$\mathbf{n}$} & \multirow{2}{*}{$\begin{array}{l}\text { Estatura/Idade } \\
\text { Média (IC 95\%) }\end{array}$} & \multirow[b]{2}{*}{$\mathbf{p}$} & \multirow{2}{*}{$\frac{\text { Peso/Idade }}{\text { Média (IC 95\%) }}$} & \multirow[b]{2}{*}{$\mathbf{p}$} & \multirow{2}{*}{$\frac{\text { IMC/Idade }}{\text { Média (IC 95\%) }}$} & \multirow[b]{2}{*}{$\mathbf{p}$} \\
\hline & & & & & & & \\
\hline Sexo da criança & & & 0,09 & & 0,34 & & 0,85 \\
\hline Masculino & 163 & $-0,49(-0,66 ;-0,32)$ & & $0,42(0,25 ; 0,60)$ & & $0,99(0,82 ; 1,17)$ & \\
\hline Feminino & 156 & $-0,21(-0,60 ; 0,17)$ & & $0,49(0,21 ; 0,78)$ & & $0,86(0,68 ; 1,04)$ & \\
\hline Idade da criança (meses) & & & 0,37 & & 0,22 & & 0,32 \\
\hline $24-60$ & 80 & $-0,41(-0,63 ;-0,19)$ & & $0,35(0,11 ; 0,59)$ & & $0,88(0,63 ; 1,13)$ & \\
\hline$<24$ & 239 & $-0,33(-0,61 ;-0,06)$ & & $0,49(0,29 ; 0,70)$ & & $0,95(0,80 ; 1,09)$ & \\
\hline Escolaridade materna & & & 0,06 & & 0,11 & & 0,52 \\
\hline $\begin{array}{l}\text { Ensino Fundamental } \\
\text { completo ou Superior }\end{array}$ & 252 & $-0,44(-0,58 ;-0,30)$ & & $0,65(0,07 ; 1,24)$ & & $0,93(0,78 ; 1,08)$ & \\
\hline $\begin{array}{l}\text { Sem instrução ou Ensino } \\
\text { Fundamental incompleto }\end{array}$ & 67 & $-0,03(-0,89 ; 0,82)$ & & $0,41(0,26 ; 0,55)$ & & $0,92(0,68 ; 1,16)$ & \\
\hline \multicolumn{2}{|c|}{ Esgotamento sanitário do domicílio } & & 0,48 & & 0,82 & & 0,28 \\
\hline Rede pública & 61 & $-0,36(-0,66 ;-0,06)$ & & $0,62(0,33 ; 0,91)$ & & $0,88(0,88 ; 1,45)$ & \\
\hline Outros & 258 & $-0,35(-0,60 ;-0,10)$ & & $0,42(0,23 ; 0,61)$ & & $0,95(0,73 ; 1.01)$ & \\
\hline \multicolumn{3}{|c|}{ Benefício do Programa Bolsa Família } & 0,65 & & 0,54 & & 0,39 \\
\hline Sim & 190 & $-0,32(-0,65 ; 0,10)$ & & $0,47(0,23 ; 0,70)$ & & $0,91(0,76 ; 1,06)$ & \\
\hline Não & 129 & $-0,41(-0,61 ;-0,21)$ & & $0,45(0,23 ; 0,66)$ & & $0,95(0,73 ; 1,17)$ & \\
\hline \multicolumn{3}{|c|}{ Completitude da equipe mínima de saúde } & 0,06 & & 0,02 & & 0,08 \\
\hline Sim & 180 & $-0,16(-0,60 ; 0,27)$ & & $0,65(0,33 ; 0,97)$ & & $1,03(0,82 ; 1,24)$ & \\
\hline Não & 139 & $-0,50(-0,67 ;-0,33)$ & & $0,31(0,15 ; 0,47)$ & & $0,85(0,70 ; 1,00)$ & \\
\hline \multicolumn{3}{|c|}{ Nutricionista na equipe de saúde } & 0,11 & & 0,04 & & 0,10 \\
\hline Sim & 162 & $-0,23(-0,60 ; 0,13)$ & & $0,59(0,32 ; 0,86)$ & & $1,01(0,82 ; 1,19)$ & \\
\hline Não & 157 & $-0,49(-0,68 ;-0,30)$ & & $0,31(0,14 ; 0,49)$ & & $0,84(0,68 ; 1,01)$ & \\
\hline \multicolumn{3}{|c|}{$\begin{array}{l}\text { Capacitação dos profissionais de saúde na área de } \\
\text { alimentação e nutrição }\end{array}$} & 0,10 & & 0,05 & & 0,09 \\
\hline Adequado & 216 & $-0,16(-0,74 ; 0,41)$ & & $0,66(0,25 ; 1,07)$ & & $1,05(0,79 ; 1,31)$ & \\
\hline Inadequado & 103 & $-0,45(-0,60 ;-0,29)$ & & $0,36(0,22 ; 0,51)$ & & $0,87(0,73 ; 1,01)$ & \\
\hline
\end{tabular}

importantes da qualidade da assistência, particularmente nos países em desenvolvimento, a qual exerce influência sobre os resultados nutricionais ${ }^{4,17}$. Nesse sentido, cabe ressaltar que as carências encontradas na completitude das equipes mínimas de saúde, na disponibilidade de diretrizes para atuar na área, na capacitação dos profissionais de saúde em nutrição e no acompanhamento do crescimento da criança vêm sendo retratada na literatura ${ }^{18-21}$. Circunstâncias como essas também representam a realidade de outros países $^{22-24}$. Apesar de que a avaliação materna dos serviços de saúde não mostrou associação com o estado nutricional das crianças, deve ser ressaltado que os atributos em análise apresentaram-se insatisfatórios, com respaldos na literatura ${ }^{5,25}$. Assim, os achados sugerem a necessidade de fortalecer o cuidado nutricional das crianças menores de cinco anos na Atenção Primária à Saúde com investimentos na formação e atuação dos profissionais de saúde e na incorporação do nutricionista nesse espaço de trabalho.

A falta de treinamento constitui um dos obstáculos relacionados às deficiências na vigilância do crescimento na rede básica de saúde no Bra$\operatorname{sil}^{18}$. A completitude da equipe de saúde em sua composição mínima e a presença do nutricionista também podem repercutir no cuidado nutricional $^{26,27}$. As associações dessa natureza encontradas nesse estudo especificamente com o peso/idade podem estar relacionadas a características desse índice como sua capacidade de expressar a situação global do estado nutricional da criança ${ }^{28}$ e seu maior uso para o monitoramento do crescimento condicionado pela inclusão na rede de serviços desde a implantação do Cartão da Criança ${ }^{29}$. 
Tabela 1. Média e intervalo de confiança de 95\% de estatura/idade, peso/idade e índice de massa corporal/ idade em escore $\mathrm{Z}$ de acordo com características sociodemográficas das crianças e dos serviços de saúde. Região Metropolitana de João Pessoa, PB, $2014(\mathrm{~N}=319)$.

\begin{tabular}{|c|c|c|c|c|c|c|c|}
\hline \multirow{2}{*}{ Variáveis } & \multirow{2}{*}{$\mathbf{n}$} & Estatura/Idade & \multirow{2}{*}{$\mathbf{p}$} & \multirow{2}{*}{$\begin{array}{c}\text { Peso/Idade } \\
\text { Média (IC 95\%) }\end{array}$} & \multirow{2}{*}{$\mathbf{p}$} & \multirow{2}{*}{$\frac{\text { IMC/Idade }}{\text { Média (IC 95\%) }}$} & \multirow{2}{*}{$\mathbf{p}$} \\
\hline & & Média (IC 95\%) & & & & & \\
\hline \multicolumn{3}{|c|}{$\begin{array}{l}\text { Disponibilidade de medicamentos e suplementos na } \\
\text { equipe de saúde }\end{array}$} & 0,52 & & 0,66 & & 0,76 \\
\hline Adequado & 270 & $-0,35(-0,60 ;-0,11)$ & & $0,47(0,29 ; 0,66)$ & & $0,95(0,81 ; 1,09)$ & \\
\hline Inadequado & 49 & $-0,37(-0,73 ;-0,01)$ & & $0,38(0,06 ; 0,69)$ & & $0,83(0,50 ; 1,16)$ & \\
\hline \multicolumn{3}{|c|}{$\begin{array}{l}\text { Disponibilidade de documentos técnicos na equipe de } \\
\text { saúde }\end{array}$} & 0,05 & & 0,05 & & 0,25 \\
\hline Adequado & 54 & $-0,29(-0,52 ;-0,05)$ & & $0,51(0,33 ; 0,69)$ & & $0,94(0,81 ; 1,08)$ & \\
\hline Inadequado & 277 & $-0,80(-1,19 ;-0,42)$ & & $0,11(0,06 ; 0,49)$ & & $0,82(0,49 ; 1,14)$ & \\
\hline \multicolumn{3}{|c|}{ Uso da Caderneta de Saúde da Criança } & 0,04 & & 0,11 & & 0,39 \\
\hline Adequado & 96 & $-0,32(-0,54 ;-0,10)$ & & $0,52(0,31 ; 0,74)$ & & $0,90(0,70 ; 1,11)$ & \\
\hline Inadequado & 223 & $-1,11(-1,82 ;-0,41)$ & & $0,30(0,07 ; 0,53)$ & & $0,94(0,78 ; 1,10)$ & \\
\hline \multicolumn{3}{|c|}{ Utilização dos serviços de saúde } & 0,18 & & 0,19 & & 0,40 \\
\hline Adequado & 184 & $-0,24(-0,69 ; 0,20)$ & & $0,54(0,22 ; 0,87)$ & & $0,91(0,75 ; 1,07)$ & \\
\hline Inadequado & 135 & $-0,44(-0,60 ;-0,27)$ & & $0,390,23 ; 0,56$ & & $0,95(0,74 ; 1,15)$ & \\
\hline \multicolumn{3}{|c|}{ Acessibilidade aos serviços de saúde } & 0,29 & & 0,44 & & 0,62 \\
\hline Adequado & 93 & $-0,32(-0,60 ;-0,03)$ & & $0,44(0,23 ; 0,65)$ & & $0,96(0,75 ; 1,17)$ & \\
\hline Inadequado & 226 & $-0,44(-0,66 ;-0,23)$ & & $0,47(0,25 ; 0,68)$ & & $0,92(0,76 ; 1,07)$ & \\
\hline \multicolumn{3}{|c|}{ Longitudinalidade dos serviços de saúde } & 0,08 & & 0,09 & & 0,22 \\
\hline Adequado & 45 & $-0,29(-0,53 ;-0,05)$ & & $0,55(0,29 ; 0,81)$ & & $0,87(0,70 ; 1,04)$ & \\
\hline Inadequado & 274 & $-0,72(-1,05 ;-0,39)$ & & $0,33(0,16 ; 0,51)$ & & $0,97(0,79 ; 1,15)$ & \\
\hline \multicolumn{3}{|c|}{ Integralidade dos serviços de saúde } & 0,21 & & 0,22 & & 0,34 \\
\hline Adequado & 77 & $-0,31(-0,57 ;-0,04)$ & & $0,49(0,29 ; 0,70)$ & & $0,88(0,66 ; 1,10)$ & \\
\hline Inadequado & 242 & $-0,50(-0,75 ;-0,25)$ & & $0,35(0,12 ; 0,57)$ & & $0,94(0,79 ; 1,09)$ & \\
\hline
\end{tabular}

Fonte: Elaborado pelos autores.

Tabela 2. Modelo de regressão linear multivariada de estatura/idade, peso/idade e índice de massa corporal em escore $\mathrm{Z}$ de acordo com características sociodemográficas das crianças e dos serviços de saúde. Região Metropolitana de João Pessoa, PB, $2014(\mathrm{~N}=319)$.

\begin{tabular}{|c|c|c|c|c|c|c|c|c|c|}
\hline \multirow[t]{2}{*}{ Variáveis $^{\star}$} & \multicolumn{3}{|c|}{ Estatura/Idade } & \multicolumn{3}{|c|}{ Peso/Idade } & \multicolumn{3}{|c|}{$\begin{array}{l}\text { Índice de Massa } \\
\text { Corporal/Idade }\end{array}$} \\
\hline & $\boldsymbol{\beta}$ & IC 95\% & p & $\boldsymbol{\beta}$ & IC 95\% & $\mathbf{p}$ & $\boldsymbol{\beta}$ & IC 95\% & $\mathbf{p}$ \\
\hline $\begin{array}{l}\text { Mãe sem instrução ou com ensino } \\
\text { fundamental incompleto }\end{array}$ & $-0,54$ & $-0,58 ;-0,09$ & 0,04 & & & - & & & - \\
\hline Equipe mínima de saúde incompleta & $-0,46$ & $-0,73 ;-0,03$ & 0,06 & 0,08 & 0,$03 ; 0,21$ & 0,02 & 0,26 & 0,$20 ; 0,36$ & 0,08 \\
\hline Equipe de saúde sem nutricionista & $-0,44$ & $-0,75 ;-0,25$ & 0,08 & 0,22 & 0,$13 ; 0,28$ & 0,03 & 0,27 & 0,$15 ; 0,33$ & 0,09 \\
\hline $\begin{array}{l}\text { Capacitação dos profissionais de } \\
\text { saúde na área de alimentação e } \\
\text { nutrição inadequada }\end{array}$ & $-0,21$ & $-0,47 ;-0,11$ & 0,09 & 0,34 & 0,$19 ; 0,44$ & 0,04 & 0,33 & 0,$30 ; 0,41$ & 0,09 \\
\hline $\begin{array}{l}\text { Uso da Caderneta de Saúde da } \\
\text { Criança inadequado }\end{array}$ & $-0,32$ & $-0,80 ;-0,08$ & 0,03 & & & - & & & - \\
\hline $\begin{array}{l}\text { Disponibilidade de documentos } \\
\text { técnicos do Ministério da Saúde na } \\
\text { equipe de saúde inadequada }\end{array}$ & $-0,48$ & $-0,60 ;-0,11$ & 0,06 & 0,19 & 0,$09 ; 0,29$ & 0,06 & & & - \\
\hline
\end{tabular}


Apesar de poucas evidências na literatura científica, relatos sobre a associação do cuidado nutricional com características da equipe de saúde, como sua composição, capacitação e inclusão de nutricionista, tem sido relatado tanto na literatura nacional ${ }^{20,30}$ quanto internacional ${ }^{22,24}$. Essa relação pode ter explicações na capacidade da qualificação na área de nutrição para o aperfeiçoamento da atenção nutricional ${ }^{31}$ e na importância do nutricionista para melhorias na estrutura e no processo de trabalho ${ }^{30}$, acarretando conscientização e corresponsabilidade entre os diferentes membros da equipe de saúde em relação às práticas de cuidado nutricional ${ }^{19}$. Esses argumentos traduzem, inclusive, a visão dos profissionais de saúde ao ressaltar a importância do nutricionista na qualificação da equipe para a atenção nutricional, segundo resultados constatados em estudo realizado no município do Rio de Janeiro. Adicionalmente, de acordo com esse mesmo trabalho, outros fatores como preferir que seja o próprio profissional nutricionista quem execute as orientações de alimentação e nutrição e considerar complexas as demandas de cuidado nutricional podem ter significados relevantes para o trabalho interdisciplinar ${ }^{32}$.

Dar ênfase às questões nutricionais como parte do treinamento contínuo dos profissionais de saúde pode fortalecer a avaliação nutricional e favorecer, portanto, a gestão da desnutrição e do tratamento das doenças comuns na infância ${ }^{33}$. Entretanto, no Brasil, as ações de alimentação e nutrição são desenvolvidas, principalmente, por médicos e enfermeiros, os quais, em geral, não apresentam a capacitação adequada para atuar na área e delegam essas ações a um segundo plano ${ }^{34}$. Assim, como discutido por outros pesquisadores, desprende-se a necessidade urgente de preparar melhor os profissionais de saúde para abordar as condições nutricionais usando as melhores práticas, processo no qual os nutricionistas devem assumir um papel de liderança ${ }^{35}$.

A associação encontrada neste estudo entre o preenchimento da CSC e a estatura/idade sugere que o crescimento da criança representa um medida sensível aos cuidados de saúde. A importância das questões de alimentação e nutrição na CSC, sendo a estatura um importante indicador da situação de saúde, pode relacionar-se a esse resultado. $\mathrm{O}$ fato da relação observada ter sido em específico para a estatura/idade é, talvez, reflexo desse índice ser o mais sensível à qualidade de vida e que melhor expressa o efeito cumulativo de situações adversas sobre o crescimento da criança ${ }^{26,28}$. Desse modo, é possível postular a importância da CSC na vigilância e promoção da saúde da criança, sobretudo em relação ao crescimento linear, corroborando achados anteriores ${ }^{36}$, o que reafirma as práticas adequadas de vigilância do crescimento como promotoras do estado nutricional das crianças ${ }^{37}$ e reforça a necessidade de investimentos que visem melhorar a utilização da CSC como instrumento de monitoramento da saúde infantil ${ }^{21}$.

Adicionalmente, cabe destacar que neste estudo a escolaridade materna foi a única condição sociodemográfica que se associou à estatura/idade da criança, confirmando a importância desta variável no estado nutricional conforme relatos recorrentes da literatura ${ }^{6,38,39}$. No Brasil, a melhoria da escolaridade materna constitui uma das razões principais que explicam o declínio do déficit de estatura nas últimas décadas, por influenciar positivamente a utilização dos recursos financeiros e dos serviços de saúde, bem como o cuidado à criança ${ }^{39}$.

Crianças beneficiárias e não beneficiárias do PBF não apresentaram diferenças nos escores $Z$ dos índices antropométricos, reflexo, possivelmente, das características dos serviços de saúde em termos de disponibilidade, qualidade e implicações na adesão às condicionalidades de saúde, que fazem a transferência de renda não produzir resultados no estado nutricional, conforme estudo de revisão que não apontou efeitos do Programa no estado nutricional dos beneficiários ${ }^{8}$. Em sintonia com esses resultados, análises baseadas em dados de âmbito nacional mostraram maiores médias de estatura/idade, peso/idade e índice de massa corporal/idade entre crianças que não recebiam o benefício do $\mathrm{PBF}^{40}$. Outro estudo contraria esses achados, estabelecendo o impacto positivo do Programa no estado nutricional das crianças de 0 a 5 anos, condicionado ao cumprimento das condicionalidades de saúde e ao acompanhamento sistemático na atenção básica em saúde ${ }^{41}$, o que ficou fora do alcance deste estudo.

A interpretação dos resultados apresentados deve considerar que os mesmos representam apenas o contexto dos serviços de saúde, de áreas de abrangência da ESF. Além disso, alguns vieses podem ter ocorrido como consequência da coleta de dados ter sido realizada nos serviços de saúde e o caráter transversal da pesquisa inviabiliza o estabelecimento de direção nas associações analisadas. Do ponto de vista do modelo teórico adotado, é importante sinalizar possíveis limitações relacionadas à não contemplação de determinantes do estado nutricional como a situação de saúde e o consumo alimentar da criança, as- 
sim como da renda familiar que contribui sobremaneira nesses aspectos.

\section{Conclusão}

Por todo o exposto, conclui-se o presente trabalho afirmando sua contribuição com o conhecimento ao indicar associações do estado nutricional da criança com características estruturais (recursos humanos) e processuais (preenchi- mento da CSC) de equipes de saúde da ESF. Estes achados são inovadores e relevantes para as políticas públicas de saúde. Deste modo, sugere-se o desenvolvimento de pesquisas sobre modelos de treinamento com foco no cuidado nutricional e seus efeitos nas práticas dos profissionais de saúde e na população. Para esses fins, deve considerar-se o desenvolvimento de habilidades relacionadas à avaliação nutricional, diagnóstico nutricional, intervenções nutricionais e monitoramento e avaliação.

\section{Colaboradores}

Figueroa Pedrazza D participou da concepção do estudo. Figueroa Pedrazza D e MM Oliveira participaram do levantamento, análise e interpretação dos dados, redação, revisão crítica e aprovação do manuscrito. Os autores declaram serem responsáveis por todos os aspectos do estudo, garantindo sua exatidão e integridade. 


\section{Referências}

1. Victora CG, Rivera JA. Optimal child growth and the double burden of malnutrition: research and programmatic implications. Am J Clin Nutr 2014; 100(Supl.):1611S-1612S.

2. Conde WL, Monteiro CA. Nutrition transition and double burden of undernutrition and excess of weight in Brazil. Am J Clin Nutr 2014; 100(Supl.):1617S $-1622 S$.

3. Miglioli TC, Fonseca VM, Gomes Junior SC, Silva KS, Lira PIC, Batista Filho M. Fatores associados ao estado nutricional de crianças menores de cinco anos. Rev Saude Publica 2015; 49:59.

4. The Lancet. Maternal and Child Nutrition. Executive Summary of The Lancet Maternal and Child Nutrition Series [cited 2018 Nov 1]. Lancet [homepage on the Internet]. Available in: https://www.thelancet. $\mathrm{com} / \mathrm{pb} / \mathrm{assets} / \mathrm{raw} /$ Lancet/stories/series/nutrition -eng.pdf

5. Leão $\mathrm{CDA}$, Caldeira $\mathrm{AP}$, Oliveira MMC. Atributos da atenção primária na assistência à saúde da criança: avaliação dos cuidadores. Rev Bras Saude Matern Infant 2011; 11(3):323-334.

6. Stewart CP, Iannotti L, Dewey KG, Michaelsen KF, Onyango AW. Contextualising complementary feeding in a broader framework for stunting prevention. Maternal Child Nut 2013; 9(Supl. 2):27-45.

7. Jaime PC, Silva ACF, Lima AMC, Bortolini GA. Ações de alimentação e nutrição na atenção básica: a experiência de organização no Governo Brasileiro. Rev Nutr 2011;24(6):809-824.

8. Wolf MR, Barros Filho AA. Estado nutricional dos beneficiários do Programa Bolsa Família no Brasil - uma revisão sistemática. Cienc Saude Colet 2014; 19(5):1331-1338.

9. Canella DS, Silva ACF, Jaime PC. Produção científica sobre nutrição no âmbito da Atenção Primária à Saúde no Brasil: uma revisão de literatura. Cienc Saude Colet 2013; 18(2):297-308.

10. Brasil. Ministério da Saúde (MS). Saúde da criança: crescimento e desenvolvimento. Brasília: MS; 2012. (Cadernos de Atenção Básica, nº 33).

11. Brasil. Ministério da Saúde (MS). Programa Nacional de Suplementação de Ferro: manual de condutas. Brasília: MS; 2013.

12. Brasil. Ministério da Saúde (MS). Manual de condutas gerais do Programa Nacional de Suplementação de Vitamina A. Brasília: MS; 2013.

13. Brasil. Ministério da Saúde (MS). Portaria no 1.498 , de 19 de julho de 2013. Redefine o Calendário Nacional de Vacinação, o Calendário Nacional de Vacinação dos Povos Indígenas e as Campanhas Nacionais de Vacinação, no âmbito do Programa Nacional de Imunizações (PNI), em todo o território nacional. Diário Oficial da União 2013; 1ª seção - p.31; 22 jul.

14. Brasil. Ministério da Saúde (MS). Manual do instrumento de avaliação da atenção primária à saúde: primary care assessment tool pcatool - Brasil. Brasília: MS; 2010.

15. World Health Organization (WHO). Physical status: the use and interpretation of anthropometry. Geneva: WHO; 1995. (WHO - Technical Report Series, 854).
16. World Health Organization (WHO). WHO Child Growth Standards. Length/height-for-age, weight-for -age, weight-for-length, weight-for-height and body mass index-for-age. Methods and development. Geneva: WHO; 2006.

17. Bacon TJ, Newton WP. Innovations in the education of health professionals. NC Med J 2014; 75(1):22-27.

18. Figueroa Pedraza D. Growth surveillance in the context of the Primary Public Healthcare Service Network in Brazil: literature review. Rev Bras Saude Matern Infant 2016;16(1):7-19.

19. Rigon AS, Schmidt ST, Bógus CM. Desafios da nutrição no Sistema Único de Saúde para construção da interface entre a saúde e a segurança alimentar e nutricional. Cad Saude Publica 2016; 32(3):e00164514.

20. Figueroa-Pedraza D. Implementation of food and nutrition actions in the context of family health strategy, Paraíba, Brazil. Rev Fac Nac Salud Publica 2019; 37(3):98-109.

21. Almeida AC, Mendes LC, Sad IR, Ramos EG, Fonseva VM, Peixoto MVM. Uso de instrumento de acompanhamento do crescimento e desenvolvimento da criança no Brasil-revisão sistemática de literatura. Rev Paul Pediatr 2016; 34(1):122-131.

22. Billah SM, Saha KK, Khan ANS, Chowdhury AH, Garnelt SP, Arifeen SE, et al. Quality of nutrition services in primary health care facilities: Implications for integrating nutrition into the health system in Bangladesh. PLoS ONE 2017; 12(5):e0178121.

23. Ghodsi D, Omidvar N, Rashidian A, Raghfar H, EiniZinab H, Ebrahimi M. Key Informants' Perceptions on the Implementation of a National Program for Improving Nutritional Status of Children in Iran. Food Nut Bull 2016; 38(1):78-91.

24. Bolka H, Abajobir AA. The practice of essential nutrition actions in healthcare deliveries of Shebedino District, South Ethiopia. Adv Reprod Sciences 2014; 2(1):8-15.

25. Damasceno SS, Nóbrega VM, Coutinho SED, Reichert APS, Toso BRGO, Collet N. Saúde da criança no Brasil: orientação da rede básica à Atenção Primária à Saúde. Cienc Saude Colet 2016;21(9):2961-2973.

26. Rocha ACD, Pedraza DF. Acompanhamento do crescimento infantil em unidades básicas de saúde da família do município de Queimadas, Paraíba, Brasil. Texto Contexto Enferm 2013; 22(4):1169-1178.

27. Laporte-Pinfildi ASC, Zangirolani LTO, Spina N, Martins PA, Medeiros MAT. Atenção nutricional no pré-natal e no puerpério: percepção dos gestores da Atenção Básica à Saúde. Rev Nutr 2016; 29(1):109123.

28. Brasil. Ministério da Saúde (MS). Orientações para a coleta e análise de dados antropométricos em serviços de saúde: Norma Técnica do Sistema de Vigilância Alimentar e Nutricional - SISVAN. Brasília: MS; 2011. (Série G. Estatística e Informação em Saúde).

29. Araújo EMN, Gouveia MTO, Pedraza DF. Use of a child health surveillance instrument focusing on growth. A cross-sectional study. Sao Paulo Med J 2017; 135(6):541-547. 
30. Machado PMO. Atenção Nutricional no âmbito da Atenção Primária à Saúde: análise de implantação em municípios brasileiros [tese]. Santa Catarina: Universidade Federal de Santa Catarina; 2018.

31. Sunguya BF, Poudel KC, Mlunde LB, Urassa DP, Yasuoka J, Jimba M. Nutrition training improves health workers' nutrition knowledge and competence to manage child undernutrition: a systematic review. Frontiers in Public Health 2013; 1:37.

32. Fittipaldi ALM, Barros DC, Romano VF. Apoio matricial nas ações de alimentação e nutrição: visão dos profissionais da Estratégia Saúde da Família. Physis 2017; 27(3):793- 811.

33. Billah SM, Saha KK, Khan ANS, Chowdhury AH, Garnett SP, Arifeen SE, Menon P. Quality of nutrition services in primary health care facilities: Implications for integrating nutrition into the health system in Bangladesh. PLoS ONE 2017; 12(5):e0178121.

34. Junqueira TS, Cotta RMM. Matriz de ações de alimentação e nutrição na Atenção Básica de Saúde: referencial para a formação do nutricionista no contexto da educação por competências. Cien Saude Colet 2014; 19(5):1459-1474.

35. Kris-Etherton PM, Akabas SR, Bales CW, Bistrian B, Braun L, Edwards MS, Laur C, Lenders CM, Levy MD, Palmer CA, Pratt CA, Ray S, Rock CL, Saltzman E, Seidner DL, Van Horn L. The need to advance nutrition education in the training of health care professionals and recommended research to evaluate implementation and effectiveness. Am J Clin Nutr 2014; 99(Supl.):1153S-1166S.

36. Figueroa Pedraz D. Preenchimento da caderneta de saúde da criança e antropometria de crianças J Manag Prim Health Care 2019;10:e10.

37. Mangasaryan N, Arabi M, Schultink W. Revisiting the concept of growth monitoring and its possible role in community-based nutrition programs. Food Nutr Bull 2011; 32(1):42-53.
38. Akombi BJ, Agho KE, Hall JJ, Wali N, Renzaho AMN, Merom D. Stunting, Wasting and Underweight in Sub-Saharan Africa: A Systematic Review. Int J Environ Res Public Health 2017; 14:863.

39. Figueroa Pedraza D. Crescimento linear das crianças brasileiras: reflexões no contexto da equidade social. Rev Nutr 2016;29(2):287-296.

40. Santos LP, Gigante DP. Relação entre insegurança alimentar e estado nutricional de crianças brasileiras menores de cinco anos. Rev Bras Epidemiol 2013;16(4):984-994.

41. Brasil. Ministério de Desenvolvimento Social e Combate à Fome (MDS). Avaliação da evolução temporal do estado nutricional das crianças de 0 a 5 anos beneficiárias do Programa Bolsa Família (PBF), acompanhadas nas condicionalidades de saúde. Brasília: MDS; 2014.

Artigo apresentado em 07/12/2019

Aprovado em 27/05/2020

Versão final apresentada em 29/05/2020

Editores-chefes: Romeu Gomes, Antônio Augusto Moura da Silva 